\title{
Traduire
}

Ine autre perspective sur I t raduction

Revue française de la traduction

245 | 2021

Regards sur l'interprétation

\section{L'interprétation à distance dans le domaine de la santé. La difficile gestion des émotions}

Daniel Secchi

\section{Q OpenEdition \\ 1 Journals}

Édition électronique

URL : https://journals.openedition.org/traduire/2444

DOI : 10.4000/traduire.2444

ISSN : 2272-9992

Éditeur

Société française des traducteurs

Édition imprimée

Date de publication : 5 décembre 2021

Pagination : 58-66

ISSN : 0395-773X

\section{Référence électronique}

Daniel Secchi, «L'interprétation à distance dans le domaine de la santé. La difficile gestion des

émotions », Traduire [En ligne], 245 | 2021, mis en ligne le 05 décembre 2021, consulté le 23 décembre 2021. URL : http://journals.openedition.org/traduire/2444 ; DOI : https://doi.org/10.4000/traduire. 2444 


\section{L'interprétation à distance dans le domaine de la santé}

La difficile gestion des émotions

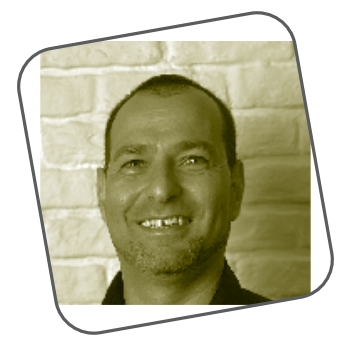

\section{Daniel Secchi}

\section{Introduction}

Lorsque nous parlons de télé-interprétation, également appelée interprétation à distance, nous faisons allusion, comme son nom l'indique, à un type spécifique d'interprétation réalisé par le biais d'appels téléphoniques ou vidéo. Ce type d'interprétation s'effectue dans différents contextes: en entreprise, dans les services publics, dans les services d'urgence, qu'il s'agisse des soins de santé et/ou des services de police. Il est à noter que le secteur qui enregistre le plus grand nombre de demandes depuis environ deux ans est celui de l'interprétation relative aux soins de santé. II convient ici de faire une distinction importante entre l'interprétation médicale sur place ou en présence, où l'interprète est physiquement présent à l'hôpital, dans une salle de consultation ou partout où sa présence est requise, et l'interprétation médicale à distance, où l'interprète effectue son travail par le biais d'appels téléphoniques ou vidéo. Ce dernier type d'interprétation à distance est le plus demandé, car il est le plus rapide à mettre en place. 
Beaucoup de choses se sont passées depuis 1973, date à laquelle l'Australie a ouvert la voie dans le domaine de l'interprétation à distance en créant, au sein du Translating and Interpreting Service, un nouveau département exclusivement chargé de l'interprétation téléphonique pour les services publics. Ce dispositif a reçu le nom de Free Emergency Telephone Interpreting Service et il avait pour objectif de fournir une assistance à tous les non-anglophones qui avaient besoin de l'aide d'un interprète dans l'un des services publics disponibles.

Depuis sa création, l'interprétation à distance s'est développée et a évolué dans le monde entier jusqu'à devenir l'une des formes d'interprétation les plus répandues. Selon Ozolins (2011: 33), "peu de domaines de l'interprétation ont connu l'impact radical de la technologie autant que l'interprétation par téléphone'». Cet impact s'explique grâce aux progrès de la technologie, qui en facilite grandement l'organisation.

En outre, depuis le début de la pandémie de Covid-19, une demande croissante d'interprètes à distance a été observée dans le monde entier dans différents secteurs, notamment celui des soins de santé. C'est dans ce contexte sociosanitaire de pandémie que les interprètes médicaux ont expérimenté de nouvelles possibilités, mais aussi de grands défis, et que la technologie dont nous disposons aujourd'hui leur a permis de se connecter virtuellement avec les patients hospitalisés par le biais d'appels téléphoniques et vidéo.

De ce fait, la plupart des interprètes médicaux, habitués à travailler sur place, ont été contraints de pratiquer à distance pour les hôpitaux - autant de nouveaux défis à relever pour les établissements de santé, les médecins, les patients et les interprètes.

Pour aborder les situations toujours plus critiques occasionnées par la pandémie, il a fallu mobiliser de nombreux professionnels de l'interprétation médicale à distance. Comme il s'agit de situations aux retombées psychologiques lourdes, nous soulignerons ici l'importance d'une bonne gestion de l'impact émotionnel, tant pour les patients que pour les

1. Traduction de l'auteur. 
interprètes médicaux, qu'ils soient expérimentés ou non, afin que ces derniers puissent fournir un service de qualité sans être affectés par le contexte.

\section{Difficultés supplémentaires dans le domaine de la santé}

Face à l'urgence sanitaire, l'interprétation à distance semble être devenue l'une des rares solutions, et les intervenants jovent un rôle clé dans la gestion des moments délicats pour les personnes ayant contracté le virus en dehors de leur pays (Rodrigo, 2020). Les interprètes doivent souvent communiquer des nouvelles difficiles, comme signaler qu'un membre de la famille a été intubé ou doit l'être, ou faire part à la famille d'un décès dont l'informe le médecin.

Ces situations, déjà très délicates à gérer pour l'interprète, prennent encore une autre ampleur en modalité à distance, générant davantage de stress et d'anxiété pour le professionnel.

Outre les difficultés techniques (qualité de la ligne téléphonique, de la connexion internet ou de l'audio), l'interprète est confronté à l'éloignement et au fait qu'il ne voit ni le médecin ni le patient. Plusieurs interprètes signalent la gêne qu'ils ressentent à communiquer avec les patients par téléphone plutôt qu'en face-à-face, car ils ne peuvent compter ni sur le langage corporel ni sur les expressions du visage (Goldberg, 2020). La transmission d'informations d'une langue à l'autre peut s'en trouver faussée et cela peut devenir une source de stress, de tension, de nervosité, voire d'un sentiment d'insécurité néfaste à la qualité du travail et au psychisme de l'interprète.

\section{Importance de la formation des interprètes médicaux à la gestion des émotions}

Le personnel de santé est formé et préparé aux situations humaines extrêmes qu'il rencontre régulièrement dans l'exercice de sa profession, alors que pour les interprètes, notamment pour ceux qui viennent d'atterrir dans le monde de l'interprétation médicale, ces aspects peuvent être totalement nouveaux, inattendus et difficiles à gérer. 
Il est donc indispensable d'intégrer dans la formation de tous les interprètes, et encore plus dans celle des interprètes médicaux, la gestion des émotions, d'un point de vue théorique, mais aussi pratique, en mettant l'accent sur l'aspect psychologique du travail. Les interprètes seraient ainsi armés pour gérer non seulement les émotions des patients dans les situations délicates, mais aussi leurs propres émotions, afin que celles-ci n'influent ni sur leur pratique ni sur leur stabilité psychologique et émotionnelle (Pöchhacker et Kadric, 1999; Trovato, 2012; Quintana et Valverde, 2021). Montanheiro (2016: 16) insiste également sur la nécessité d'une formation spécialisée en interprétation médicale et souligne que cette préparation est la clé pour que les interprètes médicaux parviennent à maîtriser la charge émotionnelle à laquelle ils sont confrontés pendant leurs heures de travail.

Compte tenu de l'urgence sanitaire mondiale actuelle, l'interprétation à distance a été un élément essentiel dans la pandémie de Covid-19. Toutefois, dans de nombreux cas, les services de santé ont fait appel à des interprètes nouvellement qualifiés ou, à défaut, à des professionnels en cours d'apprentissage.

Des auteurs tels que Wiegand (2000) et Mezcua (2010) ont déjà souligné qu'il est important pour les interprètes non seulement de connaître la terminologie spécifique, la culture de leurs interlocuteurs et de savoir se déplacer avec agilité dans leurs contextes de travail, mais aussi de savoir surmonter la douleur et le chagrin que l'état de santé précaire des patients peut éveiller en eux.

Dans le cadre de son travail, l'interprète médical, sur place ou à distance, est le premier à recevoir le message du médecin qu'il devra transmettre en maîtrisant à la fois ses propres émotions et celles de ses interlocuteurs. Des études spécifiques décrivent comment les interprètes peuvent réduire les effets de leurs émotions, afin qu'elles aient moins d'impact sur leur pratique. Valero (2006) se concentre plus précisément sur l'explication des variables (personnelles et contextuelles) qui affectent négativement les interprètes, tandis que Morales (2015) propose aux interprètes des solutions pour maîtriser leurs émotions, dont la plus basique est de ne pas s'identifier au patient et d'éviter tout lien émotionnel avec lui. 
Quoique l'interprétation à distance soit connue depuis longtemps, la pandémie a intensifié son utilisation dans les services de santé, au point de supplanter parfois l'interprétation médicale en face-à-face, car moins dangereuse dans le contexte spécifique de la Covid-19. Mezcua (2010) observe ainsi les conséquences néfastes du déclenchement d'émotions telles que le stress, la tension, la nervosité qui peuvent aller «jusqu'à la souffrance du syndrome d'épuisement professionnel ou de l'épuisement par empathie». Azarmina et Wallace (2005) mettent quant à eux en évidence une lacune dans la gestion pourtant nécessaire des émotions par les professionnels du secteur ainsi que l'importance d'une recherche future qui puisse faire la lumière sur cette composante du métier.

\section{Stratégies pour la maîtrise des émotions}

Dans la phase de formation, les interprètes doivent comprendre que ce travail peut provoquer chez eux des états de stress, d'anxiété, de dépression et de perte d'estime de soi. Ils auront alors besoin, en plus d'une préparation linguistique, culturelle et communicative, d'un apprentissage de la maîtrise de leurs émotions.

Pour cela, l'interprète doit apprendre à être émotionnellement stable, à éviter d'être impulsif et bouleversé, et éviter de trop s'impliquer dans les soucis des patients. Les techniques psychologiques susceptibles d'aider les interprètes sont les mêmes que celles recommandées par les psychologues qui traitent les travailleurs de la santé, soit, en essence:

a. la relaxation progressive avec la méthode de Jacobson (1938), qui consiste à détecter les tensions musculaires, à détendre l'ensemble de l'appareil musculaire et à relâcher certains muscles pendant la réalisation d'une tâche donnée;

b. la respiration diaphragmatique ou abdominale (Vallejo et Ferrer, 2000:157), qui consiste à inspirer et expirer lentement et profondément;

c. I'entraînement autogène avec la méthode Schultz (1980: 25), qui consiste à produire une transformation générale du sujet expérimental au moyen de certains exercices physiologiques et rationnels et 
qui, par analogie avec les plus anciennes pratiques hypnotiques exogènes, permet d'obtenir des résultats identiques à ceux obtenus avec des états suggestifs authentiques;

d. l'entraînement à la relaxation autoappliquée qui, selon Carranque (2004), comprend:

- l'autotranquillisation obtenue par la relaxation interne;

- l'autorégulation des fonctions organiques (cardiocirculatoire, respiratoire, digestive, entre autres);

- l'augmentation des performances (mémoire, etc.);

- I'autodétermination obtenue par la formulation d'objectifs spécifiques, qui permet de mieux faire face à de nombreuses situations stressantes de la vie;

- la capacité d'autocritique et de maîtrise de soi;

- la formulation de résolutions pour la réalisation d'objectifs humains éthiques;

e. la distraction cognitive, une technique qui consiste à changer volontairement, contre un autre stimulus, le centre d'attention des pensées et des sensations que la personne (en l'occurrence l'interprète) éprouve; cette technique est utile pour gérer les crises de panique et réduire l'anxiété (García et Gallego, 2003: 512).

\section{Conclusion}

L'interprétation à distance est une réalité depuis 1973, soit depuis près de cinquante ans, et ne peut donc pas être considérée comme une nouvelle forme d'interprétation. La gravité de la situation sanitaire mondiale, engendrée par la pandémie de Covid-19, a accéléré la demande d'interprètes à distance dans le domaine de la santé et a en même temps mis en évidence l'une des difficultés de cette profession, à savoirl'incapacité ou la capacité limitée à gérer correctement et efficacement les émotions et donc la santé psycho-émotionnelle de ces professionnels.

Plusieurs études liées à la gestion des émotions dans les professions de l'interprétation en personne et à distance dans les services publics ont déjà été réalisées au cours des deux dernières décennies par des auteurs tels que Wiegand (2000), Moser-Mercer (2003), Valero (2006), 
Ozolins (2011), Del Pozo Triviño (2013), Valero (2006, 2015), Morales (2015), Cordero (2016), Montanheiro (2016), Gradolí (2016), Gómez et Solís (2020) et Quintana (2021). Néanmoins, un vide reste à combler s'agissant de la pratique en milieu sanitaire, aussi bien en présentiel qu'à distance.

Consacrer une partie de la formation des futurs téléinterprètes à un point aussi important que la gestion des émotions permettrait de mieux préparer les professionnels à intervenir, y compris dans des situations d'urgence sanitaire, sans que leur travail soit excessivement affecté par le contexte. Tout le monde y gagnerait: les interprètes, les médecins et, surtout, les patients.

\section{secchi@unica.it}

Daniel SECCHI est interprète et traducteur depuis 2013 et ses langues de travail sont l'espagnol, le français et l'italien. II est titulaire d'un doctorat en études hispaniques avancées, d'un mastère en enseignement de l'espagnol comme langue étrangère et est également examinateur DELE accrédité par I'Instituto Cervantes pour tous les niveaux de A1 à C2. Après avoir exercé en France et en Espagne, il enseigne actuellement I'interprétation (espagnol/italien) et la médiation interculturelle (espagnol/italien) à I'Università degli Studi di Cagliari (Italie).

SOURCES CITÉES

AZARMINA Pejman et WALLACE Paul, «Remote Interpretation in Medical Encounters: a Systematic Review», in Journal of Telemedicine and Telecare, 2005, 11(3), p. 140-145.

CORDERO CID María et al., Prevención del síndrome "burnout" en intérpretes en los servicios públicos, Madrid, Universidad de Alcalá, 2016, https://ebuah.uah.es/ dspace/handle/10017/23651, consulté le 23/04/2021.

DEL POZO TRIVIÑO María Isabel, «Camino a la profesionalización de los intérpretes en los servicios públicos y asistenciales españoles en el siglo XXI», in Cuadernos de ALDEEU, 25, 2013, p. 109-130.

GARCÍA RAMOS Javier et GALLEGO RODRIGUEZ Jose Manuel, «Psicoterapias en atención primaria», in Terapeutica en APS, 10(7), 2003, p. 508-513. 
GOLDBERG Emma, «La atención a los pacientes del coronavirus se pierde en la traducción», The New York Times, 2020, https://www.nytimes.com/es/2020/04/17/ espanol/ciencia-y-tecnologia/interprete-coronavirus-ayuda.html, consulté le 03/04/2021.

GÓMEZ PARRILLA Laura et SOLÍS GUTIÉRREZ María del Mar, «El estrés en la Interpretación para los Servicios Públicos: Protocolo de actuación y estrategias para la formación de intérpretes », in Lebende Sprachen, 65(1), 2020, p. 104-135.

GRADOLÍ JULIÁN Clara et al., Propuesta de formación psicológica especializada para intérpretes: una aproximación preliminar, Castellón (Espagne), Universitat Jaume I, 2016, http://repositori.uji.es/xmlui/handle/10234/162039, consulté le 22/06/2021.

JACOBSON Edmundo, Relajación progresiva, Chicago, The University of Chicago, 1929.

MEZCUA RUIZ Aurora, « La enseñanza de la interpretación social en el contexto biosanitario: la preparación psicológica del intérprete médico ", in TRANS. Revista de Traductología, 14, 2010, p. 143-160.

MEZCUA RUIZ Aurora, «La interpretación en los centros sanitarios: "A veces médico, a veces paciente... pero siempre intérprete” ", in Skopos. Revista internacional de traducción e interpretación, 14, 2013, p. 185-200.

MONTANHEIRO TÁSSIA Rafaela, La formación y especialización en interpretación médico-sanitaria en contextos de violencia de género, Castellón (Espagne), Universitat Jaume I, 2016, http://repositori.uji.es/xmlui/handle/10234/166369? show=full, consulté le 27/05/2021.

MORALES MARÍN Ana, El impacto psicológico en el intérprete, Alcalá de Henares (Espagne), Universidad de Alcalá de Henares, 2015, https://ebuah.uah.es/dspace/ handle/10017/23778, consulté le 14/06/2021.

MOSER-MERCER Barbara, Remote Interpreting: Assessment of Human Factors and Performance Parameters, Joint Project International, 2003, https://aiic.org/ document/516/AIICWebzine_Summer2003_3_MOSER-MERCER, consulté le 08/02/2021.

OZOLINS Uldis, «Telephone Interpreting: Understanding Practice and Identifying Research Needs », in Translation \& Interpreting, 3(2), 2011, p. 33-47.

PÖCHHACKER Franz et KADRIC Mira, «The Hospital Cleaner as Healthcare Interpreter: A Case Study», in The Translator, 5(2), 1999, p. 161-178.

QUINTANA ROJAS Lyn Valentina et VALVERDE CASTRO Jennifer Paola, El manejo de emociones de los intérpretes en la interpretación médica remota en el contexto de la pandemia de la Covid-19 en Lima, Perú, Lima, Universidad peruana de ciencias aplicadas, 2021, https://repositorioacademico.upc.edu.pe/handle/10757/654578, consulté le 22/07/2021.

RODRIGO Anna, Intérprete de línea de emergencias: somos la voz del médico para extranjeros, EFE, 2020, https:/www.efe.com/efe/espana/destacada/interpretede-linea-emergenciassomos-la-voz-del-medico-para-extranjeros/10011-4217498, consulté le 13/01/2021.

SCHULTZ JOHANNES HEINRICH, Entrenamiento Autógeno, Barcelona (Espagne), Científico Médica, 1980. 
Translating and Interpreting Service, Australian Government Department of Home Affairs, TIS National, https://www.tisnational.gov.au/en/About-TISNational/History-of-TIS-National, consulté le 15/07/2021.

TROVATO Giuseppe, «La mediación cultural en el ámbito sanitario: algunas reflexiones relativas a las problemáticas culturales y al papel del mediador", in Redit (Revista electrónica de didáctica de la traducción y la interpretación), 2012, 8, p. 29-41.

VALERO GARCÉS M. Carmen et al., «El impacto psicológico y emocional en los intérpretes y traductores de los servicios públicos: un factor a tener en cuenta», in Quaderns. Revista de traducció, 13, 2006, p. 141-154.

VALERO GARCÉS M. Carmen, «The Impact of Emotional and Psychological Factors on Public Service Interpreters: Preliminary Studies», Translation \& Interpreting, 7(3), 2015, p. 90-102.

VALLEJO RUILOBA Julio et FERRER GASTÓ Cristóbal, Trastornos afectivos: ansiedad y depresión, Barcelona (Espagne), Masson, 2000.

WIEGAND Chriss, «Role of the Interpreter in the Healing of a Nation: An Emotional View», in The Critical Link 2, John Benjamins Publishing, 2000, p. 207. 